\title{
Primary Follicular Lymphoma of the Duodenum: A Case Report
}

\author{
Jin Roh $\cdot$ Jooryung Huh $\cdot$ Chan-Sik Park \\ Department of Pathology, Asan Medical Center, University of Ulsan College of Medicine, Seoul, Korea
}

Follicular lymphoma (FL) is a neoplasm of the germinal center B-cells and usually involves lymph nodes. Primary intestinal FL is an uncommon variant of FL. ${ }^{1}$ Among them, FL that involves the duodenum is reported separately as primary FL of the duodenum (PFL-D) for its unique indolent clinical course. PFL-D frequently involves the second portion of the duodenum, and it has been reported to have a very good prognosis even without any treatment. ${ }^{2}$ Due to its rarity and indolent behavior, unlike nodal FL, pathologists should be careful not to make under, or particularly, overdiagnosis. Recently, we experienced a case of PFL-D.

\section{CASE REPORT}

A 47-year-old male patient with no remarkable past medical history underwent gastroduodenoscopy as a part of preventive medical examination. Endoscopically, whitish multiple small mucosal nodules were noted adjacent to the major duodenal papilla. The lesion was confined to the mucosal layer (Fig. 1A, B). A biopsy was taken on suspicion of duodenal adenoma.

Microscopically, a polypoid lesion composed of a single prominent lymphoid follicle was demonstrated in the mucosa. The germinal center of the follicle mainly consisted of uniform centrocyte-like cells without tingible body macrophages. Small lymphoid cells similar to those found in the germinal center of the lesion were also present in the extrafollicular lamina propria of the villi (Fig. 1C). The submucosa was unremarkable. The tumor cells

\section{Corresponding Author}

Chan-Sik Park, MD, PhD

Department of Pathology, Asan Medical Center, University of Ulsan College

of Medicine, 88 Olympic-ro 43-gil, Songpa-gu, Seoul 05505, Korea

Tel: +82-2-3010-5838, Fax: +82-2-472-7898, E-mail: csikpark@amc.seoul.kr

Received: December 10, 2015 Revised: January 18, 2016

Accepted: January 27, 2016 were immunopositive for CD20, CD10, Bcl-6, and Bcl-2. Ki67 reactivity rate was very low (Fig. 1D, E). Notably, CD21positive follicular dendritic cells (FDC) were condensed and redistributed at the periphery of the neoplastic follicle as described before (Fig. 1F). ${ }^{3}$

The patient underwent staging work-up including laboratory tests, positron emission tomography computed tomography, and bone marrow biopsy. No evidence of systemic involvement was identified. In the process of retrospective review of the patient's medical history, we found that the patient had annually undergone gastroduodenoscopy since 2012. We re-examined the biopsy slide that had been diagnosed as benign lymphoid follicular aggregation in the past, and confirmed that the lesion was also PFL-D by immunohistochemistry. There has been no endoscopic aggravation of the lesion during the past 2 years.

\section{DISCUSSION}

In this paper, we report a case of PFL-D that is a rare variant of FL. Schmatz et al. ${ }^{2}$ retrospectively reviewed 63 cases of PFL-D for a median follow-up time of longer than 6 years. In that study, PFL-D occurred once per 3,000 to 7,000 gastroduodenoscopies and showed good prognosis even in heterogeneous treatments including watch and wait, radiation, Rituximab monotherapy, and chemotherapy. Of note, seven cases showed complete regression without any treatment. ${ }^{2}$ However, a case of high grade transformation of PFL-D was reported recently. ${ }^{4}$

Differentiation between PFL-D and reactive lymphoid hyperplasia or other B-cell lymphomas can be problematic. Normal follicles have tingible body macrophages in contrast to the neoplastic follicles in PFL-D. Centrocyte predominance lacking normal polarization is another characteristic of the neoplastic follicle. ${ }^{5}$ Strong immunopositivity for Bcl-2 and low immunopositivity 

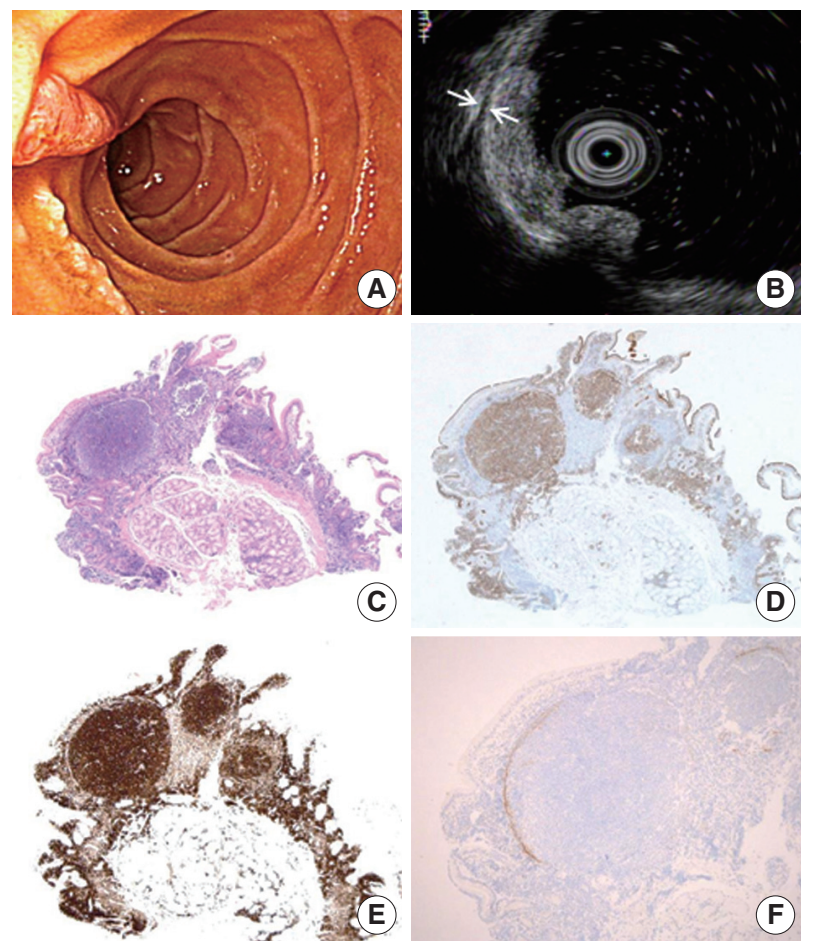

Fig. 1. Endoscopic and histopathology of primary follicular lymphoma of the duodenum. (A) Whitish multi-nodular mucosal lesions are present around major papilla. (B) A hypoechoic mucosal disruption is noted is demonstrated by endosonography. The lesion is limited to the mucosa. The muscularis propria remains intact between the two arrows. (C) Histologically, a polypoid lesion shows a prominent germinal center. This neoplastic germinal center is immunopositivity for CD10 (D) and Bcl-2 (E). Note identical FL cells are also seen in the mucosa outside germinal center. $(F)$ CD21 immunohistochemical stain highlights remaining follicular dendritic cells that are condensed in the periphery of the neoplastic germinal center.

for $\mathrm{Ki}-67$ in the neoplastic follicles in addition to CD10 and Bcl-6-positive cells in extrafollicular region are also distinguishing features of PFL-D from benign follicular hyperplasia. ${ }^{6}$ In the gastrointestinal tract, extranodal marginal zone lymphoma of mucosa-associated lymphoid tissue (MALT lymphoma) and diffuse large B-cell lymphoma (DLBCL) are the most common extranodal non-Hodgkin lymphoma. DLBCL can be differentiated from PFL-D relatively easily due to typical diffuse infiltrates of large atypical lymphoid cells. MALT lymphoma also rarely forms nodules and shows lymphoepithelial lesions. Unique endoscopic features of PFL-D, typically enlarged whitish villi or variably sized white spots, can be a distinguishing feature.

Although PFL-D has a lot in common with nodal FL regarding histologic, molecular, and immunohistochemical phenotypes, several distinguishing features have been reported. ${ }^{2}$ PFL-D has been reported that has exclusively low grade (grade 1-2) histology unlike nodal FL that shows high grade histology (grade 3) in $10 \%$ to $20 \%$ of the cases. ${ }^{1,8}$ Our case also shows histologic features of the grade 1. Another notable feature is the pattern of FDC arrangement. Meshworks of FDC in nodal FL are present in follicular areas. ${ }^{9}$ However, a majority of PFL-D including our case show severely disrupted follicular pattern as in the follicular colonization of the MALT lymphoma. CD21 or CD23 immunohistochemical stain highlights rearrangement of FDC around the periphery of the neoplastic follicles. ${ }^{2,6}$ Takata et al. ${ }^{3}$ suggested that PFL-D be considered as a distinct entity based on this specific FDC pattern in addition to activation-induced cytidine deaminase loss and $\mathrm{BACH} 2$ expression.

In summary, in duodenal multi-nodular or multiple small polypoid lesions with prominent lymphoid follicles, PFL-D should be considered as one of the differential diagnoses. To our knowledge, the present case is the first reported case of PFL-D in Korea. Making an accurate diagnosis of PFL-D is important for the proper work-up in order to avoid an aggressive over-treatment.

\section{Conflicts of Interest}

No potential conflict of interest relevant to this article was reported.

\section{REFERENCES}

1. Harris NL, Swerdlow SH, Jaffe ES, et al. Follicular lymphoma. In: Swerdlow SH, Campo E, Harris NL, et al., eds. Who classification of tumours of haematopoietic and lymphoid tissues. Lyon: IARC Press, 2008; 220-6.

2. Schmatz AI, Streubel B, Kretschmer-Chott E, et al. Primary follicular lymphoma of the duodenum is a distinct mucosal/submucosal variant of follicular lymphoma: a retrospective study of 63 cases. J Clin Oncol 2011; 29: 1445-51.

3. Takata K, Sato Y, Nakamura N, et al. Duodenal and nodal follicular lymphomas are distinct: the former lacks activation-induced cytidine deaminase and follicular dendritic cells despite ongoing somatic hypermutations. Mod Pathol 2009; 22: 940-9.

4. Miyata-Takata T, Takata K, Sato Y, et al. A case of diffuse large B-cell lymphoma transformed from primary duodenal follicular lymphoma. Pathol Int 2014; 64: 527-32.

5. Takata K, Miyata-Takata T, Sato Y, Yoshino T. Pathology of follicular lymphoma. J Clin Exp Hematop 2014; 54: 3-9.

6. Graham RL, Mardones MA, Krause JR. Primary follicular lymphoma of the duodenum. Proc (Bayl Univ Med Cent) 2015; 28: 381-3.

7. Iwamuro M, Okada H, Takata K, et al. Magnified endoscopic fea- 
tures of duodenal follicular lymphoma and other whitish lesions. Acta Med Okayama 2015; 69: 37-44.

8. Kodama M, Kitadai Y, Shishido T, et al. Primary follicular lymphoma of the gastrointestinal tract: a retrospective case series. Endoscopy 2008; 40: 343-6.
9. Zukerberg LR, Medeiros LJ, Ferry JA, Harris NL. Diffuse lowgrade B-cell lymphomas. Four clinically distinct subtypes defined by a combination of morphologic and immunophenotypic features. Am J Clin Pathol 1993; 100: 373-85. 